\title{
DTWDIR: AN ENHANCED DTW ALGORITHM FOR AUTISTIC CHILD BEHAVIOUR MONITORING
}

\author{
Salwa O Slim ${ }^{1}$, Ayman Atia ${ }^{1}$, and Mostafa-Sami M.Mostafa ${ }^{1}$ \\ ${ }^{1}$ HCI-LAB, Department of Computer Science, Faculty of Computers and Information, \\ Helwan University, Cairo, Egypt
}

\begin{abstract}
Autism has symptoms can hardly be recognized in the early stages of the disease, and it affects the child's mental health on the long term. Autism can be identified by parents monitoring to the child and diagnosed by psychiatrists using an international standard checklist. The checklist questions should be answered by the parent and psychiatrist to determine the risk level of autism (high, medium, or low risk). It is hard for parents to monitor more than 20 child's behaviours at the same time regardless lack of accuracy for answering on most of these questions. We propose a system for monitoring autistic child behaviours by analysing accelerometer data collected from wearable mobile device. The behaviours are recognized by using a novel algorithm called DTWDir that based on calculating displacement and direction between two signals. DTWDir is evaluated by comparing it to KNN, classical Dynamic Time Warping (DTW), and One Dollar Recognition (\$1) algorithms. The results show that DTWDir accuracy is higher than the others.
\end{abstract}

\section{KEYWORDS}

Autistic child, behaviours monitoring, DTW, KNN, One Dollar recognition

\section{INTRODUCTION}

According to a new report from the U.S. Centres for Disease Control and Prevention, there is a $30 \%$ increase in autistic children ratio than two years ago. Autism spectrum disorder (ASD) and autism are both general terms for a group of complex disorders of brain development. These disorders are characterized -in varying degrees- by difficulties in social interaction, verbal and nonverbal communication, and repetitive behaviours. Psychiatrist who works with children must deal with a great variety of behavioural and emotional problems therefore some of researches provided standardized assessment and documentation of such problems and requires a little effort by the psychiatrist. Those standardized assessments, may be a questionnaire [1] [2] or a checklist standard [3] [4], are filled by parents and psychiatrist. The parents have a problem answering all the questions accurately, because it requires parents' awareness to all the behaviours that result from the autistic child and monitor the child for a long time attentively. Figure1 displays script from standardized assessment Questions. 


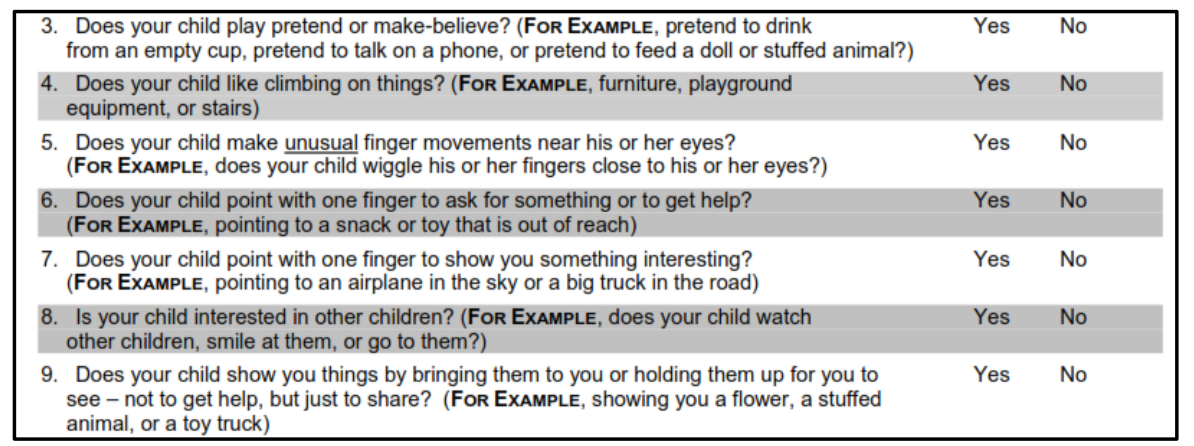

Figure 1. Script from modified checklist standard assessment for autism in toddlers [5]

The psychiatrists have to calculate the total score of the checklist questions and consequently determining the level of autism risk. If the total score is 0-2 that means low risk, 3-7 is medium risk, and 8-20 is high risk.

The majority of researches attempt to automatically identify the autistic child by monitoring repetitive behaviours rather than social and communication defects [6][7] [8] which are easily identified by using standardized assessments. This article is offering an automated methodology to answer some of the standard checklist questions. Those questions are focused on

- The ability of the child to copy what you do such as clapping like you does.

- If the child has imagination and plays pretend or make-believe such as imaging he/she drink from the cup.

- If the child does stereotypical motor movement such as flapping hand.

- And if the child interacts and communicates with the others such as acting goodbye to the others.

It is hard to monitor and collect children's behaviours let alone autistic children, therefore we have the challenge to collect data and apply an algorithm that can recognize the child's behaviour using a small dataset. We collected the four behaviours (goodbye wave, drinking, hand flapping, clapping) from a normal children by using accelerometer build in the smartphone and recognized those behaviours using a novel algorithm called DTWDir that is based on comparing signals displacement and direction. The displacement value is calculated by using DTW classifier and direction value is calculated by collecting the direction variation -the difference in signs- between two signals.

We evaluated our approach by comparing to KNN, One Dollar Recognition (\$1), and classical Dynamic Time Warping (DTW) classifiers. KNN accuracy is directly proportional the size of the dataset even if the accuracy change is not effective [24]. Many researches depended on reducing the size of datasets [25], [26], [27], and [28] when it applied KNN, as it has to use all the training examples on each test which increases execution time with large datasets [29][30][31]. Bagnal et al. [32] determined that 1-NN with an elastic measure such as DTW is the best approach for smaller data sets, but that as the number of series increases "the accuracy of elastic measures converges with that of Euclidean distance". Euclidian distance calculation is the square of the difference between two points and that means the value's sign are lost therefore we developed DTWDir which considers the absolute distance value and also its sign. By comparing DTWDir to KNN, \$1, and DTW the results showed that DTWDir accuracy is higher than the others and reached to $93 \%$ when using three axes together. 
The rest of the paper is as follows: we review related work in section2. Section3 explains the DTWDir classifier. Dataset is explained in section 4. The experiments and results are explained in section5. Section6 include the Discussions. Conclusion is explained in section7.

\section{RELATED WORK}

The related work is divided into two parts the first one is related to some of the classifiers that are used for recognizing living activities and the second part displays the previous works which are related to the autistic child.

\subsection{Living Activities}

Many researchers monitored the living activities by using smart phone accelerometer. Sang et al. [9] and Ortiz et al. [10] have proposed a system to monitor some of living activities by using KNN for recognition and they compared it with others classifiers. The authors found that KNN accuracy reach to 74\%, 88\% in [9],[10] respectively. Sang et al. found that the recognition results of Driving, sitting, and On-table activities reach to $100 \%$ but the results of Downstairs and upstairs activities are poor due to the ambiguity of accelerometer and gyroscope data therefore the authors suggests in future that those two activities should be concerned as a single activity for better recognition results.

Paiyarom et al. [18] recognized ten activities of daily living by using DTW classifier. The data are collected from two subjects - female, male- and they found that the mean accuracy of DTW is $91 \%$ even though the dataset training size is small. Muscillo et al. [19] compared between decision tree C4.5 and DTW classifiers for recognizing low-level food preparation activities. The data are collected from four sensors equipped kitchen utensils (knifes and spoon) about 6 hours. They found that DTW accuracy is better than C4.5. Li et al. [20] developed a new algorithm depends on using cubic spline interpolation and Derivative DTW by using Derivative DTW classifier on the interpolated original data. They compared between DTW, Derivative DTW, and the new algorithm on twelve different datasets and found that the accuracy of the new algorithm is beneficial when DTW could not recognize the specific dataset and can produce much less number of warping. Nayak et al. [21] proposed a new algorithm that is similar to the algorithm of the Li et al. [20] but it used DTW classifier rather than Derivative DTW on the interpolated original data. The authors used five different probabilistic distance measurements for DTW and they evaluated the new algorithm on three different datasets; sign recognition (with large number of possible classes), gesture recognition (with person variations), and classification of human interaction sequences (with segmentation problems). The results showed that DTW without reduction dimensions (original data) is better than DTW with reduction (interpolation).

\subsection{Autistic Child}

Most of the previous researches interest was to monitor stereotypical motor movements to identify the autistic child and did not care about the interaction and communication of the child with others such as Westeyn et al. [8] and Albinali et al. [6]. Westeyn et al. used 3-axes accelerometers for detecting stereotypical motor movements from individuals mimicking the actual behaviours. They achieved 69\% of hand flapping behaviour, which automatically and accurately is detected by using Hidden Markov Model, when Insertion errors are reduced.

Albinali et al. published two articles. Albinali et al. 2009 collected the data from 3-axes accelerometer readings from 6 autistic children. They used J48 Decision Tree classifier, which is an open source Java implementation of the decision tree algorithm $(\mathrm{C} 4.5)$ in the WEKA data mining tool, to identify stereotypical motor movements in the classroom and laboratory and they 
found the mean accuracy is $88.6 \%, 89.5 \%$ respectively [7]. A major concern is the high false positive rates averaging 0.08 across all participants. For intervention applications that target specific stereotypical motor movements, the system would incorrectly deliver the intervention $8 \%$ of the time when the participant is not engaged in the behaviour. Albinali et al. 2012 added expert and non-experts system using software on mobile phones to annotate stereotypical motor movements for classifier training [6]. They didn't collect any sensor data related to child's interaction and communication.

Chuah et al. [11] developed Smartphone-Based Autism Social Alert (SASA) for automatically detecting autistic child's stereotypical behaviours early and send alert to caregivers. They recognized audio background with the occurrence of stereotypical behaviours so as to identify potential environmental factors that may trigger such behaviours. Chuah et al. also used the same algorithm (J48 Decision Tree classifier) of the Albinali et al 2009 and 2012 but Chuah et al. distinguished stereotypical behaviours like foot tapping, jumping, hand waving, walking, sitting with an accuracy of $85 \%$.

Postawka et al. [22] used kinect sensor and the skeleton structure for monitoring the autistic child. HMM algorithm is used for activity modelling and emotions recognition. They applied experiments on standing and sitting activities. Gonçalves et al. [23] aimed to identify if the Kinect sensor and the eZ430-Chronos watch with the accelerometers can be used as a time-efficient tool to automatic detect child's behaviours. Two behaviours (hand flapping and body rocking movement) are collected from four autistic children by using Kinect sensor and wireless accelerometers placed on each wrist and chest. They used DTW recognizer and found that DTW accuracy when using accelerometers sensors $(76 \%)$ is better than when using kinect sensor $(51 \%)$.

\section{DTWDir ClaSSIFIER}

The DTWDir is an improvement to DTW classifier. DTW is a technique for matching two time series' data [15]. The main purpose is to align two series in an optimal way, i.e. minimizing costs for the warping path [16]. This path can be found very efficiently using dynamic programming to evaluate the following recurrence which defines the cumulative distance $\alpha\left(\mathrm{x}_{\mathrm{i}}, \mathrm{y}_{\mathrm{j}}\right)$ as the Euclidian distance $d\left(x_{i}, y_{j}\right)$ found in the current cell and the minimum of the cumulative distances of the adjacent elements:

$$
\begin{gathered}
\alpha(i, j)=d\left(x_{i}, y_{j}\right)+\min \left(\alpha\left(x_{i-1}, y_{j}\right), \alpha\left(x_{i}, y_{j-1}\right), \alpha\left(x_{i-1}, y_{j-1}\right)\right) \\
d\left(x_{i}, y_{j}\right)=\left(x_{i}-y_{j}\right)^{2} .
\end{gathered}
$$

DTWDir depends on two main measured values on the selected Window length. These values are the displacement value which is calculated by DTW classifier and the direction value which aim to find the sign variation between the two series.

The following example illustrates the different between KNN, DTW, and DTWDir classifiers on three synthetic signals (Temp1, Temp2, Query) for X-axis, Y-axis in Figure 2.

We can classify Query as Temp 1 series because the directions of both signals in $\mathrm{X}$-axis are (positive $(+\mathrm{Ve})$ then negative $(-\mathrm{Ne}))$ but in Temp2 are $(-\mathrm{Ne})$ then $(+\mathrm{Ve})$. In the Y-Axis three signals are the same. 

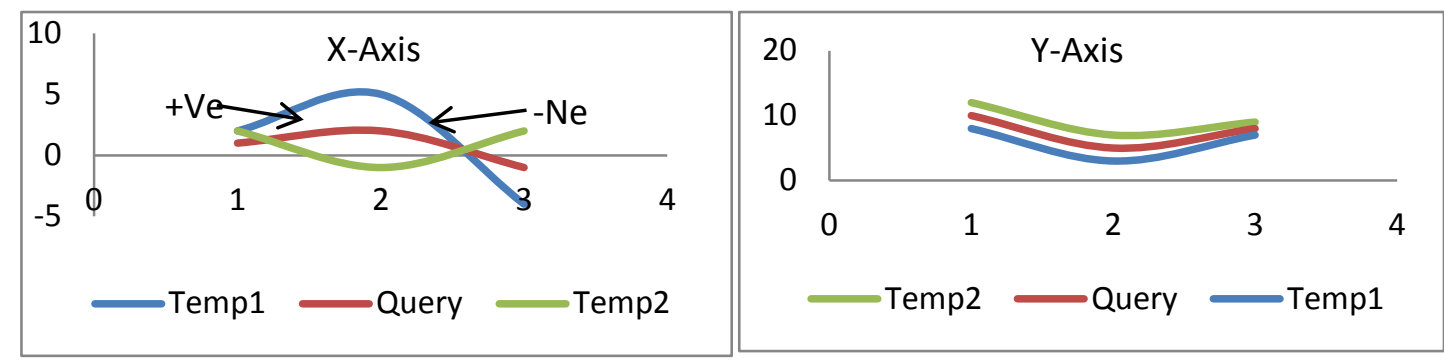

Figure 2. Three synthetic signals in $(\mathrm{X}, \mathrm{Y})$ axes

In KNN, Query signal is classified as Temp1 or Temp2 because the displacement of Query to Temp1 and Temp2 are the same. DTW classified Query as Temp2 series because it measured the displacement between Query and Temp2 $(\approx 15.87)$ is less than the displacement between Query and Temp1 $(\approx 17.25)$.DTWDir was able to classify Query as Temp1 series because its number of sign variation is smaller than Temp2.

\section{DTWDir Pseudo Code}

Given Testing Template $\mathrm{Q}$ that has sequence of points $\mathrm{Q}=\mathrm{p}_{\mathrm{q} 1} \cdots \mathrm{p}_{\mathrm{qn}}$, where $\mathrm{n}=$ number of samples(Q)

Given Training Templates $T=t_{1} \cdots t_{M}$ with labels $L=l_{1}, \cdots, l_{M}$ respectively and each training template has sequence of points $t_{i}=p_{i 1} \cdots p_{i m}$, where $M$ is number of training templates and $\mathrm{im}=$ number of samples $\left(\mathrm{t}_{\mathrm{i}}\right)$

Given Window Length WL (with seconds)

Given Overlap (with seconds)

Output $l_{i}$ where $t_{i}$ is the closet template for the $Q$

//preparing the testing template by Selecting a period of time equal to WL in the center of $\mathrm{Q}$ period

$\mathrm{SWL}=\mathrm{WL} \times$ Sampling rate

SOL $=$ Overlap $\times$ Sampling rate

$\grave{\mathrm{Q}}=\mathrm{p}_{\mathrm{qi}} \cdots \mathrm{p}_{\mathrm{q}(\mathrm{i}+\mathrm{SWL})}$, where $\mathrm{i}=(\mathrm{n} / 2)-(\mathrm{SWL} / 2)$

//comparing each training template with testing template $\mathrm{Q}$

For $\mathrm{i}=1$ to $\mathrm{M}$ Do Loop

//comparing one training template $t_{i}$ with $Q$

For $j=1$ to $j+S W L \leq$ number of samples $\left(t_{i}\right)$ Do Loop

$\grave{t}_{1}=p_{i j} \cdots p_{i(j+S W L)}$

$\operatorname{arr}_{\text {dist\&dir}} \cdot$ pushback(DTWAlgorithm( $\left(t_{1}, Q\right)$, Direction $\left.\left(t_{1}, Q\right)\right)$

$\mathrm{j}=\mathrm{j}+\mathrm{SOL}$

END Loop

Ranking $\operatorname{arr}_{\text {dist\&dir }}$ with direction ascending order

TrainingResultsArrr.pushback $\left(\mathrm{t}_{\mathrm{i}}, \operatorname{arr}_{\text {dist\&dir }}[0]\right)$

End Loop

Ranking TrainingResultsArr with Distance ascending order

$\mathrm{l}_{\mathrm{i}}=$ Label of (TrainingResultsArr [0])

\section{DATA SeT}

Our data set is collected by 'Lenovo' smart phone sensor which its sampling rate is approximately 98 samples per second. We recorded $6 \mathrm{sec}$ for each try of four behaviours (goodbye, hand 
flapping, drinking, and clapping) from 3 children with ages $4 \pm 1$. We collected 10 tries per behaviour per child. The acceleration and orientation values are collected for each try per behaviour.

\section{EXPERIMENTS AND RESULTS 5.1 Experiments Setup}

A C++ application was developed to compare DTWDir with KNN, One Dollar recognizer, and DTW classifiers. K-fold cross validation with $\mathrm{K}=3$ is used to split data into 3 parts that each part is one child but before any manipulation we used high-pass filter to remove gravity from the measured acceleration [17]. As the frequency of gravity is low, it is considered that filtering low frequency acceleration is effective in reducing the impact of gravity. There are many criteria effects on DTWDir quality that are in the following list.

- Sensor type: we recorded the acceleration and the orientation data.

- Pre-processing operations: we have 3 ways for entering the data to a novel algorithm;

- Raw data: without any selection or changing in the data. This attribute isn't available to apply on KNN algorithm, because all training and testing templates should have the same length.

- Interpolation: it is applied on the raw data to ensure all templates have the same length [12].

- Selected 5 sec: it's like raw data but to guarantee the number of sampling in all templates are equal, we selected $5 \mathrm{sec}$ from the originally recorded $6 \mathrm{sec}$ of raw data.

- Axis type: there are three axes. We need to know which axis is better than the others.

As we mentioned before we aim to measure the accuracy of DTWDir by comparing with three others classifiers but there are many criteria affects on our algorithm therefore we are going to apply changes on those criteria and observe how the DTWDir will react. We summarized experiments description and goals in Table 1.

Table 1: summarized experiments analysis

\begin{tabular}{|l|l|l|}
\hline Exp & Description & Goals \\
\hline$\# 1$ & $\begin{array}{l}\text { Comparison between KNN, } \\
\text { DTW, \$1, and DTWDir using } \\
\text { the values of the sensor's axis } \\
\text { X, Y, and Z individually and } \\
\text { the three axes together using } \\
\text { two types of sensors } \\
\text { acceleration and orientation. }\end{array}$ & $\begin{array}{l}\text { 1. } \begin{array}{l}\text { Measure the accuracy of each algorithm } \\
\text { when sensor types are Orientation (OR) or } \\
\text { Acceleration (AC). }\end{array} \\
\text { Identify the best sensor for each algorithm. } \\
\text { 2.termine which axis is better than the } \\
\text { others in AC and OR sensors. }\end{array}$ \\
\hline $\begin{array}{l}\text { Comparison between four } \\
\text { algorithms when pre- } \\
\text { processing operations are } \\
\text { changed. }\end{array}$ & $\begin{array}{l}\text { 1. } \begin{array}{l}\text { Measure the accuracy of each algorithm } \\
\text { when the pre-processing operation is } \\
\text { changed }\end{array} \\
\text { Determine which pre-processing operation is } \\
\text { the best for increasing the algorithms } \\
\text { accuracy. }\end{array}$ \\
\hline
\end{tabular}




\begin{tabular}{|l|l|l|}
\hline \#3 & $\begin{array}{l}\text { Comparison between four } \\
\text { algorithms when we selected 5 } \\
\text { sec from raw data and used } \\
\text { both the three axes together } \\
\text { and the Z axis alone. }\end{array}$ & $\begin{array}{l}\text { 1. Measure the accuracy of the behaviours in } \\
\text { each algorithm. }\end{array}$ \\
\hline
\end{tabular}

\subsection{Results}

In Experiment \#1, we interpolate the raw data to 300 samples in the pre-processing operation and put the value of overlap with $0.5 \mathrm{sec}$ and window length with $2 \mathrm{sec}$. The results are shown on Table 2.

Table 2: The comparison results accuracy of four classifiers according to two sensors types (OR and AC) for each axis individually and three axes together

\begin{tabular}{|c|c|c|c|c|c|c|c|c|c|c|}
\hline \multirow[b]{2}{*}{$\begin{array}{l}\text { Sensor } \\
\text { Type } \\
\text { Classifier }\end{array}$} & \multicolumn{5}{|c|}{ OR } & \multicolumn{5}{|c|}{$\mathbf{A C}$} \\
\hline & $\mathbf{X}$ & $\mathbf{Y}$ & $\mathbf{Z}$ & $\mathbf{X}, \mathbf{Y}, \mathbf{Z}$ & AVG & $\mathbf{X}$ & $\mathbf{Y}$ & $\mathbf{Z}$ & $\mathbf{X}, \mathbf{Y}, \mathbf{Z}$ & AVG \\
\hline KNN & 69 & 74 & 62 & 80 & 71.25 & 63 & 60 & 54.6 & 58 & 58.9 \\
\hline$\$ 1$ & 39.6 & 37.3 & 59 & 49.6 & 46.3 & 36.3 & 47.3 & 35.3 & 59.6 & 44.6 \\
\hline DTW & 80.6 & 84.6 & 77 & 90 & 83 & 64 & 83 & 59.6 & 90.6 & 74.3 \\
\hline DTWDir & 83.3 & 88.6 & 86 & 89 & 86.7 & 82 & 87.3 & 64 & 90.6 & 80.9 \\
\hline & 68.1 & 71.1 & 71 & 77.1 & & 61.3 & 69.4 & 53.3 & 74.9 & \\
\hline
\end{tabular}

In Experiment \#2, we used raw data in pre-processing step, accelerometer sensor type, and Xaxis. Because raw data doesn't have the same length in all tries, KNN couldn't apply on raw data attributes. The results are shown on the Table 3.

Table 3: The accuracy of four classifiers when pre-processing operation is changed

\begin{tabular}{|c|c|c|c|}
\hline $\begin{array}{ll}\text { Entering data } \\
\text { Classifier }\end{array}$ & Raw data & $\begin{array}{l}\text { Interpolation } \\
\mathrm{N}=300\end{array}$ & Selected 5 secs \\
\hline KNN & & $63 \%$ & $60.6 \%$ \\
\hline DTW & $76 \%$ & $64 \%$ & $75.6 \%$ \\
\hline DTWDir & $80 \%$ & $82.3 \%$ & $84.6 \%$ \\
\hline$\$ 1$ & $34.6 \%$ & $36.6 \%$ & $37.6 \%$ \\
\hline Average & $63.5 \%$ & $61.4 \%$ & $64.6 \%$ \\
\hline
\end{tabular}

In Experiment \#3, we used Acceleration sensor type, overlap value is $0.25 \mathrm{sec}$ and window length value is $2 \mathrm{sec}$. the results are shown on the Table 4 . 
Table 4: Accuracy of each behaviour

\begin{tabular}{|c|c|c|c|c|c|c|c|c|}
\hline \multirow[b]{2}{*}{$\begin{array}{l}\text { Classifiers } \\
\text { Behaviours }\end{array}$} & \multicolumn{2}{|l|}{ KNN } & \multicolumn{2}{|l|}{$\$ 1$} & \multicolumn{2}{|l|}{ DTW } & \multicolumn{2}{|c|}{ DTWDir } \\
\hline & 3 Axes & Z-Axis & 3 Axes & $\begin{array}{l}\text { Z- } \\
\text { Axis }\end{array}$ & 3 Axes & Z-Axis & 3 Axes & Z-Axis \\
\hline Clapping & $100 \%$ & $100 \%$ & $46.6 \%$ & $36.6 \%$ & $100 \%$ & $83.3 \%$ & $100 \%$ & $86.6 \%$ \\
\hline Drinking & $100 \%$ & $100 \%$ & $90 \%$ & $93.3 \%$ & $100 \%$ & $73.3 \%$ & $100 \%$ & $96.3 \%$ \\
\hline Flapping & $20 \%$ & $20 \%$ & $33.3 \%$ & $13.3 \%$ & $66.6 \%$ & $3.3 \%$ & $73.3 \%$ & $33.3 \%$ \\
\hline Goodbye & $23.3 \%$ & $23.3 \%$ & $13.3 \%$ & $23.3 \%$ & $100 \%$ & $80 \%$ & $100 \%$ & $73.3 \%$ \\
\hline Average & $61 \%$ & $61 \%$ & $46 \%$ & $42 \%$ & $91.6 \%$ & $60 \%$ & $93 \%$ & $72 \%$ \\
\hline
\end{tabular}

\section{DISCUSSIONS}

We applied KNN [9] on our dataset and we found that DTWDir is better than KNN as displayed in Table 2. DTWDir is higher than KNN in all axis in both acceleration and orientation sensors. Table 2 displayed that all algorithms accuracy in the orientation data sensor is better than the acceleration data. It's better to use three axes together rather than single axis for all algorithms in both acceleration and orientation sensors. The accuracy of DTWDir in both sensor types is higher than the others.

The interpolation pre-processing may change the original data values (raw data) but it keeps the form of the signal pattern [13] therefore DTW accuracy in interpolation is less than in raw data attributes as in Table 3 because the purpose of DTW algorithm matches the best wrapping path between the two signals [14] but the DTWDir accuracy was not affected. DTW accuracy in 5 sec is better than in interpolation but it still was not higher than raw data pre-processing. The authors [20] [21] developed new algorithms depend on interpolation pre-processing and DTW classifier. The article [20] displayed that the new algorithm accuracy was not beneficial except in the case of DTW failure to recognize the behaviour and also [21] showed that DTW with raw data is better than DTW with interpolation pre-processing. The selected $5 \mathrm{sec}$ pre-processing is better than the others in all algorithms except for KNN because it depends on Euclidian distance in calculation. For sure the speed of algorithms in the interpolation is higher than the others pre-processing operations because the length of data signals in the interpolation is 300 points and in the selection $5 \mathrm{sec}$ pre-processing operation is 490 points $\left(5^{*} 98\right)$ where 98 is the sampling rate. DTWDir is better than the other algorithms in all pre-processing operations.

Most of previous researches [6] [7] [11] used decision tree algorithm (C4.5) for recognizing stereotypical motor movements to identify the autistic child but Muscillo et al. [19] displayed that DTW is better than C4.5 when they compared between them. As mentioned before, stereotypical motor movements are not enough to identify the autistic child therefore they were not in consideration, however we compared hand flapping detection accuracy in both cases when DTWDir achieved highest, and lowest accuracy using three axes together, and Z-Axis respectively. Table 2 displayed that the accuracy of algorithms in three axes together is better than the others but Z-axis is the least one. Table 4 showed that DTWDir is better than KNN, $\$ 1$ and DTW in both cases. Westeyn et al. [8] achieved 69\% by using Hidden Markov Model algorithm and a 3 axes accelerometer, but DTWDir was able to detect hand flapping behaviour with percentage $73.3 \%$. 


\section{CONCLUSION}

Psychiatrists are interested in the child's interaction and communication with the others not only stereotypical motor movement behaviours. They used checklist standard assessment to determine the risk level of autism. The checklist contains several yes or no questions each question aims to measure some of child's behaviour such as (interaction with the others, communication skills, and stereotypical motor behaviours). Those questions have to be answered by parents and psychiatrists but it is hard for the parents to monitor the child for a long time. Psychiatrists do a lot of effort to diagnose the children as they have a variety of behavioural and emotional problems. This paper will help them by providing an automated technique to answer some of the checklist questions. The questions in consideration are answered by evaluating child's behaviours which are monitored by accelerometer. We collected four behaviours; three behaviours are dealing with interaction and communication (goodbye, drinking, clapping), one behaviour is dealing with stereotypical movement (hand flapping). The dataset is recorded from three child's behaviours that mimic the autistic child. We applied KNN, \$1 recognition, and DTW classifiers on our dataset but we found that the average accuracy of three algorithms are $71 \%, 46 \%, 83 \%$ respectively using orientation sensor type and $58.9 \%, 44.6 \%, 74 \%$ respectively using acceleration sensor type. DTW is better than $\$ 1$ and KNN in both acceleration and orientation sensor types therefore we attempt to improve the accuracy of DTW recognition by developing a novel algorithm based on DTW that is called DTWDir. We evaluated the novel algorithm by comparing it to KNN, \$1 and DTW on our dataset. From the previous experiments we found that DTWDir is better than the other algorithms but it consumes high execution time. In the future work we will try to reduce the execution time by combining KNN with DTWDir.

\section{References}

[1] M. Posserud, A. Lundervold and C. Gillberg, "Autistic features in a total population of 7-9-yearold children assessed by the ASSQ (Autism Spectrum Screening Questionnaire)", J Child Psychol \& Psychiat, vol. 47, no. 2, pp. 167-175, 2006.

[2] M. Mattila, K. Jussila, S. Linna, M. Kielinen, R. Bloigu, S. Kuusikko-Gauffin, L. Joskitt, H. Ebeling, T. Hurtig and I. Moilanen, "Validation of the Finnish Autism Spectrum Screening Questionnaire (ASSQ) for Clinical Settings and Total Population Screening", J Autism Dev Disord, vol. 42, no. 10, pp. 2162-2180, 2012.

[3] Validation of the Modified Checklist for Autism in Toddlers, Revised With Follow-up (M-CHATR/F)", PEDIATRICS, vol. 133, no. 1, pp. X5-X5, 2013.

[4] Sunita and J. Bilszta, "Early identification of autism: A comparison of the Checklist for Autism in Toddlers and the Modified Checklist for Autism in Toddlers", Journal of Paediatrics and Child Health, vol. 49, no. 6, pp. 438-444, 2012.

[5] J. M. Kleinman et al., "The modified checklist for autism in toddlers: A follow-up study investigating the early detection of autism spectrum disorders," Journal of Autism and Developmental Disorders, vol. 38, no. 5, pp. 827-839, Sep. 2008.

[6] F. Albinali, M. Goodwin and S. Intille, "Detecting stereotypical motor movements in the classroom using accelerometry and pattern recognition algorithms", Pervasive and Mobile Computing, vol. 8, no. 1, pp. 103-114, 2012.

[7] Fahd Albinali, Matthew S. Goodwin, and Stephen S. Intille. "Recognizing stereotypical motor movements in the laboratory and classroom: a case study with children on the autism spectrum", In Proceedings of the 11th international conference on Ubiquitous computing (UbiComp '09). ACM, New York, pp.71-80, 2009.

[8] T. Westeyn, K. Vadas, X. Bian, T. Starner and G. D. Abowd, "Recognizing mimicked autistic self-stimulatory behaviours using HMMs," Wearable Computers, 2005. Proceedings. Ninth IEEE International Symposium on, pp. 164-167, 2005. 
[9] V. Sang, N. Thang, V. Van Toi, N. Hoang and T. Khoa, "Human Activity Recognition and Monitoring Using Smartphones", IFMBE Proceedings, vol. 46, pp. 481-485, 2015.

[10] J. Ortiz, "Smartphone-based human activity recognition", Springer International Publishing, 2015.

[11] M. Chuah and M. Diblasio. "Smartphone Based Autism Social Alert System," Mobile Ad-hoc and Sensor Networks (MSN), 2012 Eighth International Conference on, Chengdu, pp. 6-13, 2012.

[12] Wobbrock, J.O., Wilson, A.D. and Li, Y. "Gestures without libraries, toolkits or training: A \$1 recognizer for user interface prototypes", Proceedings of the ACM Symposium on User Interface Software and Technology (UIST '07). Newport, Rhode Island New York: ACM Press, pp. 159168, 2007.

[13] G. Opfer, "Gary D. Knott, Interpolating Cubic Splines", Journal of Approximation Theory, vol. 112, no. 2, pp. 319-321, 2001.

[14] H. Ding, G. Trajcevski, P. Scheuermann, X. Wang and E. Keogh, "Querying and mining of time series data", Proc. VLDB Endow., vol. 1, no. 2, pp. 1542-1552, 2008.

[15] C. Myers and L. Rabiner, "A Comparative Study of Several Dynamic Time-Warping Algorithms for Connected-Word Recognition", Bell System Technical Journal, vol. 60, no. 7, pp. 1389-1409, 1981.

[16] M. Eamonn J. Keogh, "Derivative Dynamic Time Warping", In First SIAM International Conference on Data Mining (SDM'2001), 2001.

[17] D. Goehl and D. Sachs. "Motion sensors gaining inertia with popular consumer electronics". White Paper, IvenSense Inc, 2007.

[18] S. Paiyarom, P. Tungamchit, R. Keinprasit and P. Kayasith, "Activity monitoring system using Dynamic Time Warping for the elderly and disabled people", 2009 2nd International Conference on Computer, Control and Communication, pp. 1-4, 2009.

[19] R. Muscillo, S. Conforto, M. Schmid, P. Caselli and T. D'Alessio, "Classification of Motor Activities through Derivative Dynamic Time Warping applied on Accelerometer Data", 2007 29th Annual International Conference of the IEEE Engineering in Medicine and Biology Society, pp. 4930-4933, 2007.

[20] H. Li, X. Wan, Y. Liang and S. Gao, "Dynamic Time Warping Based on Cubic Spline Interpolation for Time Series Data Mining," Data Mining Workshop (ICDMW), 2014 IEEE International Conference on, Shenzhen, pp. 19-26, 2014.

[21] S. Nayak, S. Sarkar and B. Loeding, "Distribution-Based Dimensionality Reduction Applied to Articulated Motion Recognition", IEEE Transactions on Pattern Analysis and Machine Intelligence, vol. 31, no. 5, pp. 795-810, 2009.

[22] A. Postawka and P. Śliwiński, "Recognition and Modeling of Atypical Children Behavior", Artificial Intelligence and Soft Computing, vol. 9119, pp. 757-767, 2015.

[23] N. Gonçalves, J. L. Rodrigues, S. Costa and F. Soares, "Automatic detection of stereotyped hand flapping movements: Two different approaches," RO-MAN, 2012 IEEE, Paris, pp. 392-397, 2012.

[24] S. Yu, S. Guo and S. Publishing, "Big Data Concepts, Theories, and Applications | Shui Yu | Springer", Springer.com, $2016 . \quad$ [Online]. Available: http://www.springer.com/la/book/9783319277615.

[25] P. Hart, "The Condensed Nearest Neighbour Rule," IEEE Transactions on Information Theory, vol. 14, pp. 515-516, 1968.

[26] G. Gates, "The Reduced Nearest Neighbour Rule," IEEE Transactions on Information Theory, vol. 18, pp. 431-433, 1972.

[27] M. Kubat and M. Jr, "Voting Nearest-Neighbour Subclassifiers," in Proceedings of the $17^{\text {th }}$ International Conference on Machine Learning, ICML-2000, Stanford, CA, pp. 503-510, 2000.

[28] D. R. Wilson and T. R. Martinez, "Reduction Techniques for Exemplar-Based Learning Algorithms," Machine learning, vol. 38, no. 3, pp. 257-286, 2000.

[29] G. Guo, H. Wang, D. Bell, Y. Bi, and K. Greer, "KNN Model-Based Approach in Classification," Lecture Notes in Computer Science, vol. 2888, pp. 986-996, 2003.

[30] N. Bhatia and A. Vandana,"Survey of Nearest Neighbor Techniques", (IJCSIS) International Journal of Computer Science and Information Security, vol. 8, no. 2, pp. 302-305, 2010.

[31] A. Basheer, M. Ali, G. Awad, and A. Ali, "Solving the Problem of the K Parameter in the KNN Classifier Using an Ensemble Learning Approach", ( IJCSIS) International Journal of Computer Science and Information Security, Vol. 12, No. 8, August 2014 .

[32] A. Bagnall, L. Davis, J. Hills, and J. Lines, "Transformation Based Ensembles for Time Series Classification", Proceedings of the 12th (SIAM) International Conference on Data Mining, pp. 307-319, 2012. 
International Journal of UbiComp (IJU), Vol.7, No.2, April 2016 